\title{
Tight Complexity Lower Bounds for Integer Linear Programming with Few Constraints
}

\author{
DUŠAN KNOP, Czech Technical University in Prague \\ MICHAŁ PILIPCZUK, University of Warsaw \\ MARCIN WROCHNA, University of Warsaw and University of Oxford
}

We consider the standard ILP FEASIBILITY problem: given an integer linear program of the form $\{A \mathbf{x}=\mathbf{b}, \mathbf{x} \geqslant$ $0\}$, where $A$ is an integer matrix with $k$ rows and $\ell$ columns, $\mathbf{x}$ is a vector of $\ell$ variables, and $\mathbf{b}$ is a vector of $k$ integers, we ask whether there exists $\mathbf{x} \in \mathbb{N}^{\ell}$ that satisfies $A \mathbf{x}=\mathbf{b}$. Each row of $A$ specifies one linear constraint on $\mathbf{x}$; our goal is to study the complexity of ILP FEASIBILITY when both $k$, the number of constraints, and $\|A\|_{\infty}$, the largest absolute value of an entry in $A$, are small.

Papadimitriou was the first to give a fixed-parameter algorithm for ILP FEASIBILITY under parameterization by the number of constraints that runs in time $\left(\left(\|A\|_{\infty}+\|b\|_{\infty}\right) \cdot k\right)^{O\left(k^{2}\right)}$. This was very recently improved by Eisenbrand and Weismantel, who used the Steinitz lemma to design an algorithm with running time $\left(k\|A\|_{\infty}\right) O(k) \cdot \log \|\mathbf{b}\|_{\infty}$, which was subsequently refined by Jansen and Rohwedder to $O\left(\sqrt{k}\|A\|_{\infty}\right)^{k}$. $\log \left(\|A\|_{\infty}+\|\mathbf{b}\|_{\infty}\right) \cdot \log \|A\|_{\infty}$. We prove that for $\{0,1\}$-matrices $A$, the running time of the algorithm of Eisenbrand and Weismantel is probably optimal: an algorithm with running time $2^{o(k \log k)} \cdot\left(\ell+\|\mathbf{b}\|_{\infty}\right)^{o(k)}$ would contradict the exponential time hypothesis. This improves previous non-tight lower bounds of Fomin et al.

We then consider integer linear programs that may have many constraints, but they need to be structured in a "shallow" way. Precisely, we consider the parameter dual treedepth of the matrix $A$, denoted $\operatorname{td}_{D}(A)$, which is the treedepth of the graph over the rows of $A$, where two rows are adjacent if in some column they simultaneously contain a non-zero entry. It was recently shown by Koutecký et al. that ILP FEASIBILITy can be solved in time $\|A\|_{\infty}^{2}{ }_{\infty}^{O\left(\mathrm{td}_{D}(A)\right)} \cdot\left(k+\ell+\log \|\mathbf{b}\|_{\infty}\right)^{O(1)}$. We present a streamlined proof of this fact and prove that, again, this running time is probably optimal: even assuming that all entries of $A$ and $\mathbf{b}$ are in $\{-1,0,1\}$,

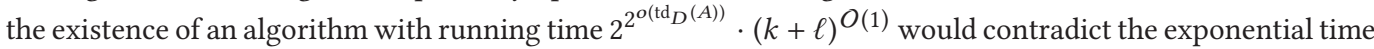
hypothesis.

CCS Concepts: • Theory of computation $\rightarrow$ Fixed parameter tractability; Integer programming;

This work is a part of projects CUTACOMBS, PowAlgDO (M. Wrochna), and TOTAL (M. Pilipczuk), which have received funding from the European Research Council (ERC) under the European Union's Horizon 2020 research and innovation programme (grant agreements 714704, 714532, and 677651, respectively). The authors acknowledge the support of the OP VVV MEYS funded project CZ.02.1.01/0.0/0.0/16_019/0000765 "Research Center for Informatics." D. Knop was supported by DFG, project “MaMu," NI 369/19. M. Wrochna was s by the Foundation for Polish Science (FNP) via the START stipend programme.

Authors' addresses: D. Knop, Department of Theoretical Computer Science, Faculty of Information Technology, Czech Technical University in Prague, Jugoslávských partyzánů 1580/3, 16000 Prague 6 - Dejvice, Czech Republic; email: dusan. knop@fit.cvut.cz; M. Pilipczuk, Institute of Informatics, University of Warsaw, Banacha 2, 02-097 Warsaw, Poland; email: michal.pilipczuk@mimuw.edu.pl; M. Wrochna, Institute of Informatics, University of Warsaw, Warsaw, Banacha 2, 02-097 Warsaw, Poland, and University of Oxford, Department of Computer Science, University of Oxford, Wolfson Building, Parks Road, Oxford, OX1 3QD, UK; email: m.wrochna@mimuw.edu.pl.

This work is licensed under a Creative Commons Attribution-NonCommercial International 4.0 License. (c) 2020 Copyright held by the owner/author(s).

1942-3454/2020/05-ART19

https://doi.org/10.1145/3397484 
Additional Key Words and Phrases: Integer linear programming, fixed-parameter tractability, ETH

ACM Reference format:

Dušan Knop, Michał Pilipczuk, and Marcin Wrochna. 2020. Tight Complexity Lower Bounds for Integer Linear Programming with Few Constraints. ACM Trans. Comput. Theory 12, 3, Article 19 (May 2020), 19 pages. https://doi.org/10.1145/3397484

\section{INTRODUCTION}

Integer linear programming (ILP) is a powerful technique used in countless algorithmic results of theoretical importance, and is applied routinely in thousands of instances of practical computational problems every day. Despite the problem being NP-hard in general, practical ILP solvers excel in solving real-life instances with thousands of variables and constraints. This can be partly explained by applying a variety of subroutines, often based on heuristic approaches, that identify and exploit structure in the input to apply the best-suited algorithmic strategies. A theoretical explanation of this phenomenon would of course be hard to formulate, but one approach is to use the paradigm of parameterized complexity. Namely, the idea is to design algorithms that perform efficiently when certain relevant structural parameters of the input have moderate values.

In this direction, probably the most significant is the classic result of Lenstra [31], who proved that ILP OPTIMIZATION is fixed-parameter tractable when parameterized by the number of variables $\ell$. In other words, it can be solved in time $f(\ell) \cdot|I|^{O(1)}$, where $f$ is some function and $|I|$ is the total bitsize of the input; we shall use this notation throughout the article. Subsequent work in this direction $[16,25]$ improved the dependence of the running time on $\ell$ to $f(\ell) \leqslant 2^{O} O(\ell \log \ell)$.

In this work, we turn to a different structural aspect and study ILPs that have few constraints, as opposed to few variables as in the setting considered by Lenstra. Formally, we consider the parameterization by $k$, the number of constraints (rows of the input matrix $A$ ), and $\|A\|_{\infty}$, the maximum absolute value over all entries in $A$. The situation when the number of constraints is significantly smaller than the number of variables appears naturally in many relevant settings. For instance, to encode SUbSET SUm as an instance of ILP FeAsibility, it suffices to introduce a $\{0,1\}$ variable $x_{i}$ for every input number $s_{i}$, and then set only one constraint: $\sum_{i=1}^{n} s_{i} x_{i}=t$, where $t$ is the target value. Note that the fact that SuBSET Sum is NP-hard for the binary encoding of the input and polynomial-time solvable for the unary encoding explains why $\|A\|_{\infty}$ is also a relevant parameter for the complexity of the problem. Integer linear programs with few constraints and many variables arise most often in the study of knapsack-like and scheduling problems via the concept of so-called configuration ILPs, in the context of approximation and parameterized algorithms.

Parameterization by the number of constraints. Probably the first to study the complexity of ILP with few constraints was Papadimitriou [36], who already in 1981 observed the following. Consider an ILP of the standard form $\{A \mathbf{x}=\mathbf{b}, \mathbf{x} \geqslant 0\}$, where $A$ is an integer matrix with $k$ rows (constraints) and $\ell$ columns (variables), $\mathbf{x}$ is a vector of integer variables, and $\mathbf{b}$ is a vector of integers. Papadimitriou proved that assuming such an ILP is feasible, it admits a solution with all variables bounded by $B=\ell \cdot\left(\left(\|A\|_{\infty}+\|\mathbf{b}\|_{\infty}\right) \cdot k\right)^{2 k+1}$, which in turn can be found in time $O\left((\ell B)^{k+1} \cdot|I|\right)$ using simple dynamic programming. Noting that by removing duplicate columns one can assume that $\ell \leqslant\left(2\|A\|_{\infty}+1\right)^{k}$, this yields an algorithm with running time $\left(\left(\|A\|_{\infty}+\|\mathbf{b}\|_{\infty}\right) \cdot k\right)^{O\left(k^{2}\right)}$. The approach can be lifted to give an algorithm with a similar running time bound also for the ILP Optimization problem, where instead of finding any feasible solution $\mathbf{x}$, we look for one that maximizes the value $\mathbf{w}^{\top} \mathbf{x}$ for a given optimization goal vector $\mathbf{w}$. 
The result of Papadimitriou [36] was recently improved by Eisenbrand and Weismantel [14], who used the Steinitz lemma to give an amazingly elegant algorithm solving the ILP OPTIMIZATION problem (and thus also the ILP FEAsibiLity problem) for a given instance $\left\{\max \mathbf{w}^{\top} \mathbf{x}: A \mathbf{x}=\mathbf{b}, \mathbf{x} \geqslant\right.$ $0\}$ with $k$ constraints in time $\left(k\|A\|_{\infty}\right)^{O(k)} \cdot \log \|\mathbf{b}\|_{\infty}$ (here, the $\log \|\mathbf{b}\|_{\infty}$ factor comes from running the LP relaxation to apply proximity bounds). This running time has been subsequently refined by Jansen and Rohwedder [24] to $O\left(\sqrt{k}\|A\|_{\infty}\right)^{2 k} \cdot \log \|\mathbf{b}\|_{\infty}$ in the case of ILP Optimization, and to $O\left(\sqrt{k}\|A\|_{\infty}\right)^{k} \cdot \log \left(\|\mathbf{b}\|_{\infty}+\|A\|_{\infty}\right) \cdot \log \|A\|_{\infty}$ in the case of ILP FEAsibility. ${ }^{1}$ They also explain how, using a proximity theorem by Eisenbrand and Weismantel [14], ILP FEASIBILITY can be solved in time $O\left(\sqrt{k}\|A\|_{\infty}\right)^{k} \cdot \log ^{2}\|A\|_{\infty}$ plus the time to solve the fractional relaxation.

From the point of view of fine-grained parameterized complexity, this raises the question of whether the parametric factor $O\left(k\|A\|_{\infty}\right)^{k}$ is the best possible. Jansen and Rohwedder [24] studied this question under the assumption that $k$ is a fixed constant and $\|A\|_{\infty}$ is the relevant parameter. They proved that assuming the strong exponential time hypothesis (SETH), for each fixed $k$ there is no algorithm with running time $\left(\|A\|_{\infty}+\|\mathbf{b}\|_{\infty}\right)^{k-\delta} \cdot|I|^{O(1)}$, for any $\delta>0$. Note that as $k$ is considered a fixed constant, this essentially shows that the degree of $\|A\|_{\infty}$ needs to be at least $k$ but does not exclude algorithms with running time of the form ${ }^{2}\|A\|_{\infty}^{O(k)} \cdot|I|^{O(1)}$, or $2^{O(k)} \cdot|I|^{O(1)}$ when all entries in the input matrix $A$ are in $\{-1,0,1\}$. However, the algorithms of Eisenbrand and Weismantel [14] and Jansen and Rohwedder [24] provide only an upper bound of $2^{O(k \log k)} \cdot|I|^{O(1)}$ in the latter setting. As observed by Fomin et al. [15], a trivial encoding of 3SAT as an ILP shows a lower bound of $2^{o(k)} \cdot|I|^{O(1)}$ for instances with $A$ having entries only in $\{0,1\}$, b having entries only in $\{0,1,2,3\}$, and $\ell=O(k)$, assuming the exponential time hypothesis (ETH). This still leaves a significant gap between the $2^{o(k)} \cdot|I|^{O(1)}$ lower bound and the $2^{O(k \log k)} \cdot|I|^{O(1)}$ upper bound.

Parameterization by the dual treedepth. A related, recent line of research concerns ILPs that may have many constraints, but these constraints need to be somehow organized in a structured, "shallow" way. It started with a result of Hemmecke et al. [20], who gave a fixed-parameter tractable algorithm for solving the so-called $n$-fold ILPs. An $n$-fold ILP is an ILP where the constraint matrix is of the form

$$
A=\left(\begin{array}{cccc}
B & B & \ldots & B \\
C & 0 & \ldots & 0 \\
0 & C & \ldots & 0 \\
\vdots & \vdots & \ddots & \vdots \\
0 & 0 & \ldots & C
\end{array}\right)
$$

and the considered parameters are the dimensions of matrices $B$ and $C$, as well as $\|A\|_{\infty}$. The running time obtained by Hemmecke et al. [20] is $\left(k\|A\|_{\infty}\right)^{O\left(k^{3}\right)} \cdot|I|^{O(1)}$ when all of these dimensions are bounded by $k$. See Hemmecke et al. [20] and the recent improvements of Eisenbrand et al. [13] for more refined running time bounds expressed in terms of particular dimensions.

The result of Hemmecke et al. [20] quickly led to multiple improvements in the best-known upper bounds for several parameterized problems, where the technique of configuration ILPs is applicable [27-29]. Recently, the technique was also applied to improve the running times of several approximation schemes for scheduling problems [23]. Chen and Marx [8] introduced a more general concept of tree-fold ILPs, where the "star-like" structure of an $n$-fold ILP is generalized to

\footnotetext{
${ }^{1}$ Throughout, log denotes the binary logarithm. The improvement from $k$ to $\sqrt{k}$ in Janson and Rohwedder [24] was obtained after the conference version; it is currently discussed only in the arXiv version [24, Theorems 9 and 10].

${ }^{2}$ Throughout this article, we will write $\|A\|_{\infty}^{O(k)}$ as a shorthand for $O\left(\|A\|_{\infty}\right)^{O(k)}$. This of course only matters when $\|A\|_{\infty}=1$.
} 
any bounded-depth rooted tree, and they showed that it retains relevant fixed-parameter tractability results. This idea was followed on by Eisenbrand et al. [12] and by Koutecký et al. [30] (see also Eisenbrand et al. [13] for a joint version), whose further generalizations apply to a structural parameter called the dual treedepth of the input matrix $A$. This parameter, denoted $\operatorname{td}_{D}(A)$, is the smallest number $h$ such that the rows of $A$ can be organized into a rooted forest of height $h$ with the following property: whenever two rows have non-zero entries in the same column, one is the ancestor of the other in the forest. As shown by the authors, ILP OptimizATion can be solved in fixed-parameter time when parameterized by $\|A\|_{\infty}$ and $\operatorname{td}_{D}(A)$.

Other structural parameters. Very recently, Chan et al. [6] showed that algorithms parameterized by dual treedepth can be extended to the parameter "branch-depth" defined on the column matroid of the constraint matrix. This parameter has the advantage of being invariant under row operations. However, the transformation incurs an exponential blow-up in the parameter. Naturally, primal treedepth (involving columns in place of rows) has also been considered, as we discuss in Section 4. Dvořák et al. [10] considered "fracture backdoors," which generalize $n$-fold ILPs and are generalized by bounded treedepth. Ganian et al. [18] and Eiben et al. [11] considered treewidth and cliquewidth instead, assuming additional bounds on coefficients and variable domains. For more detailed discussion of algorithmic implications and theory of block-structured integer programs, we refer the reader to a recent survey [7].

Our results. For the parameterization by the number of constraints $k$, we close the preceding complexity gap by proving the following optimality result.

THEOREM 1. Assuming ETH, there is no algorithm solving ILP FEASIBILITY instances $\{A \mathbf{x}=\mathbf{b}, \mathbf{x} \geqslant$ $0\}$ with $A \in\{0,1\}^{k \times \ell}, \mathbf{b} \in \mathbb{N}^{k}$, and $\ell,\|\mathbf{b}\|_{\infty}=O(k \log k)$ in time $2^{o(k \log k)}$.

This shows that the algorithms of Eisenbrand and Weismantel [14] and Jansen and Rohwedder [24] have the essentially optimal running time of $2^{O(k \log k)} \cdot|I|^{O(1)}$ in the regime where $\|A\|_{\infty}$ is a constant and the number of constraints $k$ is the relevant parameter. We can also reduce the coefficients in the target vector $\mathbf{b}$ to constant at the cost of adding negative entries to $A$.

COROLLARY 2. Assuming ETH, there is no algorithm solving ILP FEASIBILITY instances $\{A \mathbf{x}=\mathbf{b}, \mathbf{x} \geqslant$ $0\}$ with $A \in\{-1,0,1\}^{k \times \ell}, \mathbf{b} \in\{0,1\}^{k}$, and $\ell=O(k \log k)$ in time $2^{o(k \log k)}$.

The same cannot be done for non-negative matrices $A$, since in this case the algorithm of $\mathrm{Pa}$ padimitriou [36] is even simpler and works in time $\|\mathbf{b}\|_{\infty}^{O(k)} \cdot|I|^{O(1)}$ (it suffices to enumerate possible vectors $A \mathbf{x}$ such that $\|A \mathbf{x}\|_{\infty} \leqslant\|\mathbf{b}\|_{\infty}$ by dynamic programming). The reduction in Theorem 1 is hence simultaneously tight against this algorithm (since for $\|\mathbf{b}\|_{\infty}=O(k \log k)$, the bound $\|\mathbf{b}\|_{\infty}^{O(k)}$ is $\left.2^{O(k \log k)}\right)$.

The main ingredient of the proof of Theorem 1 is a certain quaint combinatorial constructiondetecting matrices introduced by Lindström [32]-that provides a general way for compressing a system $A \mathbf{x}=\mathbf{b}$ with $k$ equalities and bounded targets $\|\mathbf{b}\|_{\infty} \leqslant d$ into $O\left(k / \log _{d} k\right)$ equalities (with unbounded targets). Each new equality is a linear combination of the original ones; in fact, just taking $O\left(k / \log _{d} k\right)$ sums of random subsets of the original equalities suffices, but we also provide a deterministic construction taking $O\left(d k / \log _{d} k\right)$ such subsets. By composing such a compression procedure for $d=4$ with a standard reduction from $(3,4)$ SAT - a variant of 3SAT where every variable occurs at most four times-to ILP FEASIBILITY, we obtain a reduction that, given an instance of $(3,4)$ SAT with $n$ variables and $m$ clauses, produces an equivalent instance of ILP FEASIBILITY with $k=O((n+m) / \log (n+m))$ constraints. Since $2^{o(k \log k)}=2^{o(n+m)}$, we would obtain a $2^{o(n+m)}$-time algorithm for $(3,4)$ SAT, which is known to contradict ETH. We note that detecting matrices were recently used by two of the authors in the context of different lower bounds based on ETH [3]. 
For the parameterization by the dual treedepth, we first streamline the presentation of the approach of Koutecký et al. [30] and clarify that the parametric factor in the running time is doubly exponential in the treedepth. The key ingredient here is the upper bound on $\ell_{1}$-norms of the elements of the Graver basis of the input matrix $A$, expressed in terms of $\|A\|_{\infty}$ and $\operatorname{td}_{D}(A)$. Using standard textbook bounds for Graver bases and the recursive definition of treedepth, we prove that these $\ell_{1}$-norms can be bounded by $\left(2\|A\|_{\infty}+1\right)^{2^{\text {td }^{(A)}}-1}$. This, combined with the machinery developed by Koutecký et al. [30], implies the following.

Theorem 3. There is an algorithm that solves any given ILP Optimization instance $I=$ $\left\{\max \mathbf{w}^{\top} \mathbf{x}: A \mathbf{x}=\mathbf{b}, \mathbf{l} \leqslant \mathbf{x} \leqslant \mathbf{u}\right\}$ in time $\|A\|_{\infty}^{2^{O\left(\mathrm{td}_{D}(A)\right)}} \cdot|I|^{O(1)}$.

We remark that the running time as outlined previously also follows from a fine analysis of the reasoning presented in Koutecký et al. [30], but the intermediate step of using tree-fold ILPs in Koutecký et al. [30] makes tracking parametric dependencies harder to follow.

We next show that, perhaps somewhat surprisingly, the running time provided by Theorem 3 is optimal. Namely, we have the following lower bound.

THEOREM 4. Assuming ETH, there is no algorithm that would solve any ILP FEASIBILITY instance

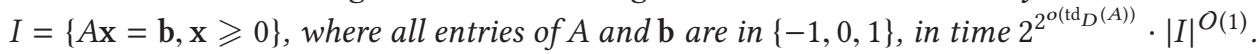

To prove Theorem 4, we reduce from the SubSET Sum problem. The key idea is that we are able to "encode" any positive integer $s$ using an ILP with dual treedepth $O(\log \log s)$. The same reduction applies also to other ranges of parameters, such as large $\|A\|_{\infty}$ and constant treedepth: see Theorem 16. This lower bound has been recently generalized by Eisenbrand et al. [13] to include a parameter they call topological height, using essentially the same reduction.

\section{PARAMETERIZATION BY THE NUMBER OF CONSTRAINTS}

\subsection{Detecting Matrices}

Our main tool is the usage of so-called detecting matrices, first studied by Lindström [32]. They can be explained via the following coin-weighing puzzle: given $m$ coins with weights in $\{0,1, \ldots, d-$ 1 , we want the deduce the weight of each coin with as few weighings as possible. We have a spring scale, so in one weighing we can exactly determine the sum of weights of any subset of the coins. Although the naive strategy-weigh coins one by one-yields $m$ weighings, it is actually possible to find a solution using $O\left(m / \log _{d} m\right)$ weighings. This number is asymptotically optimal, as each weighing provides $\Theta(\log m)$ bits of information, so fewer weighings would not be enough to distinguish all $d^{m}$ possible weight functions.

Probably the easiest way to construct such a strategy is using the probabilistic method. It turns out that querying $O\left(m / \log _{d} m\right)$ random subsets of coins with high probability provides enough information to determine the weight of each coin. This is because a random subset distinguishes any of the $O\left(d^{m} \cdot d^{m}\right)$ non-equal pairs of weight functions with probability at least $\frac{1}{2}$, but pairs of weight functions that are close to each other are few, whereas pairs of weight functions that are far from each other have a significantly better probability than $\frac{1}{2}$ of being distinguished. Note that thus we construct a non-adaptive strategy: the subsets of coins to be weighed can be determined and fixed at the very start. For an example, we refer the reader to Grebinski and Kucherov [19, Corollary 2] for full details, and we remark that the last two authors recently used detecting matrices in the context of algorithmic lower bounds for the MulTicoloRing problem [3].

Viewing each tuple of coin weights as a vector $\mathbf{v} \in\{0, \ldots, d-1\}^{m}$, each weighing returns the value $\mathbf{a}^{\top} \mathbf{v}$ for the characteristic vector $\mathbf{a} \in\{0,1\}^{m}$ of some subset of coins. Thus, $k$ weighings give the vectors of values $M \mathbf{v}$ for some $\{0,1\}$-matrix $M$ with $k$ rows and $m$ columns. An equivalent 
formulation is then to ask for a $\{0,1\}$-matrix $M$ with $m$ columns such that knowing the vector $M \mathbf{v}$ uniquely determines any $\mathbf{v} \in\{0, \ldots, d-1\}^{m}$. Such an $M$ is called a d-detecting matrix, and we seek to minimize the number of rows/weighings $k$ it can have. Lindström [32] gave a deterministic construction and proved the bound on $k$ to be tight. See also Bshouty [4] for a more direct and general construction using Fourier analysis.

TheOREM 5 [32]. For all $d, m \geqslant 2$, there is a $\{0,1\}$-matrix $M$ with $m$ columns and $k \leqslant \frac{2 m \log d}{\log m}(1+$ $o(1))$ rows such that for any $\mathbf{u}, \mathbf{v} \in\{0, \ldots, d-1\}^{m}$, if $M \mathbf{u}=M \mathbf{v}$, then $\mathbf{u}=\mathbf{v}$. Moreover, such a matrix $M$ can be constructed in time polynomial in $\mathrm{dm}$.

In other words, this allows us to check $m$ equalities between values in $\{0, \ldots, d-1\}$ (i.e., corresponding coordinates of vectors $\mathbf{u}$ and $\mathbf{v})$ using only $O\left(m / \log _{d} m\right)$ comparisons of sums of certain subsets of these values (i.e., coordinates of vectors $M \mathbf{u}$ and $M \mathbf{v}$ ). For an ILP instance $A \mathbf{x}=\mathbf{b}$ with $\|\mathbf{b}\|_{\infty} \leqslant d$ and $m$ constraints, we may use this idea to check the equality on each of the $m$ coordinates of $A \mathbf{x}$ using only $O\left(m / \log _{d} m\right)$ constraints. Indeed, the intuition is that if $M$ is a $d$ detecting matrix, then we can rewrite $A \mathbf{x}=\mathbf{b}$ as $M A \mathbf{x}=M \mathbf{b}$ and check the latter-which involves $O\left(m / \log _{d} m\right)\{0,1\}$-combinations of the original constraints (at the cost of increasing $\|A\|_{\infty},\|\mathbf{b}\|_{\infty}$ to $\left.\|M A\|_{\infty},\|M \mathbf{b}\|_{\infty}\right)$.

This is the core of our approach. However, there is one subtle caveat: to claim that the assertions $A \mathbf{x}=\mathbf{b}$ and $M A \mathbf{x}=M \mathbf{b}$ are equivalent, we would need to ensure that $\|A \mathbf{x}\|_{\infty} \leqslant d$ for an arbitrary vector $\mathbf{x} \in \mathbb{N}^{n}$. One solution is to use the fact that a uniformly random $\{0,1\}$-matrix has a stronger "detecting" property: it will, with high probability, distinguish all vectors of low $\ell_{1}$-norm, as shown by Grebinski and Kucherov [19].

LEMma 6 [19]. For all $d \geqslant 1, m \geqslant 2$, there exists a $\{0,1\}$-matrix $M$ with $m$ columns and $k \leqslant$ $\frac{4 m \log (d+1)}{\log m}(1+o(1))$ rows such that for any $\mathbf{u}, \mathbf{v} \in \mathbb{N}^{m}$ satisfying $\|\mathbf{u}\|_{1},\|\mathbf{v}\|_{1} \leqslant d m$, if $M \mathbf{u}=M \mathbf{v}$, then $\mathbf{u}=\mathbf{v}$. Moreover, such a matrix $M$ can be computed in randomized polynomial time (in $\mathrm{dm}$ ).

Note that in Lemma 6, we do not actually have to assume bounds on one of the two vectors: it suffices to assume $\mathbf{u} \in \mathbb{N}^{m}$ and $\|\mathbf{v}\|_{1} \leqslant d m$, because simply adding a single row full of ones to $M$ guarantees $\|\mathbf{u}\|_{1}=\|\mathbf{v}\|_{1}$. Therefore, as long as $A$ is non-negative and $\|\mathbf{b}\|_{\infty} \leqslant d$, it suffices to check $M A \mathbf{x}=M \mathrm{~b}$. Unfortunately, to the best of our knowledge, no deterministic construction is known for Lemma 6. We remark that Bshouty gave a deterministic but adaptive detecting strategy [4]that is, in terms of coin weighing, consecutive queries on coins may depend on results of previous weighings.

Instead, we show that a different, recursive construction by Cantor and Mills [5] for 2-detecting matrices can be adapted so that no bounds (other than non-negativity) are assumed for one of the vectors, whereas the other must have all coefficients in $\{0,1, \ldots, d-1\}$.

LEMMA 7. For all $d, m \geqslant 2$, there exists a $\{0,1\}$-matrix $M$ with $m$ columns and $k \leqslant \frac{m d \log d}{\log m}(1+$ $o(1))$ rows such that for any $\mathbf{u} \in \mathbb{N}^{m}$ and $\mathbf{v} \in\{0,1, \ldots, d-1\}^{m}$, if $M \mathbf{u}=M \mathbf{v}$, then $\mathbf{u}=\mathbf{v}$. Moreover, such a matrix $M$ can be computed in time polynomial in $d m$.

Proof of Lemma 7. Fix $d \geqslant 2$. We construct inductively for each $i \in \mathbb{N}$ a certain $\{0,1\}$-matrix $M_{i}$ with $k_{i}$ rows and $m_{i}$ columns such that for any $\mathbf{u} \in \mathbb{N}^{m_{i}}$ and $\mathbf{v} \in\{0,1, \ldots, d-1\}^{m_{i}}$, if $M \mathbf{u}=M \mathbf{v}$, then $\mathbf{u}=\mathbf{v}$. For $i=1$, we use the $d \times d$ identity matrix-that is, $k_{1}=m_{1}=d$. 
Let $B=M_{i}$ be such a matrix for $i \geqslant 1$. We claim that the following matrix $M=M_{i+1}$ with $k_{i+1}=$ $d \cdot k_{i}+d$ rows and $m_{i+1}=d \cdot m_{i}+k_{i}$ columns satisfies the same condition.

$$
M=\left(\begin{array}{ccccc}
B & B & \cdots & B & I \\
B & J-B & & & \\
\vdots & & \ddots & & \\
B & & & J-B & \\
\hline & 1 \ldots 1 & & & \\
& & \ddots & & \\
& & & 1 \ldots 1 &
\end{array}\right)
$$

Here, $I$ is the $k_{i} \times k_{i}$ identity matrix, $J$ is the $k_{i} \times m_{i}$ matrix with all entries equal to 1 , and all empty blocks are 0 . Take any $\mathbf{u} \in \mathbb{N}^{m_{i+1}}$ and $\mathbf{v} \in\{0,1, \ldots, d-1\}^{m_{i+1}}$, and write

$$
\begin{aligned}
\mathbf{u}^{\top} & =\left(\mathbf{x}^{(1) \top}|\cdots| \mathbf{x}^{(d) \top} \mid \mathbf{z}^{\top}\right) \text { for } \mathbf{x}^{(1)}, \ldots, \mathbf{x}^{(d)} \in \mathbb{N}^{m_{i}}, \mathbf{z} \in \mathbb{N}^{k_{i}} ; \\
\mathbf{v}^{\top} & =\left(\mathbf{x}^{\prime(1)^{\top}}|\cdots| \mathbf{x}^{\prime(d) \top} \mid \mathbf{z}^{\prime \top}\right) \text { for } \mathbf{x}^{\prime(1)}, \ldots, \mathbf{x}^{\prime(d)} \in\{0, \ldots, d-1\}^{m_{i}}, \mathbf{z}^{\prime} \in\{0, \ldots, d-1\}^{k_{i}} .
\end{aligned}
$$

Then, $M \mathbf{u}=M \mathbf{v}$ is equivalent to the following.

$$
\begin{array}{rlrl}
\sum_{i=1}^{d} B \mathbf{x}^{(i)}+I \mathbf{z} & =\sum_{i=1}^{d} B \mathbf{x}^{(i)}+I \mathbf{z}^{\prime} & \\
B \mathbf{x}^{(1)}+(J-B) \mathbf{x}^{(i)} & =B \mathbf{x}^{\prime(1)}+(J-B) \mathbf{x}^{\prime(i)} & & (i=2 \ldots d) \\
\sum_{j=1}^{m_{i}} x_{j}^{(i)} & =\sum_{j=1}^{m_{i}} x_{j}^{\prime(i)} & (i=2 \ldots d) \\
\sum_{j=1}^{k_{i}} z_{j} & =\sum_{j=1}^{k_{i}} z_{j}^{\prime} &
\end{array}
$$

Equation (3) is equivalent to $J \mathbf{x}^{(i)}=J \mathbf{x}^{\prime(i)}$ (for $i=2 \ldots d$ ), thus the sum of (1) with all (2) equations implies $d B \mathbf{x}^{(1)}+\mathbf{z}=d B \mathbf{x}^{\prime(1)}+\mathbf{z}^{\prime}$ and hence $z_{j} \equiv z_{j}^{\prime}(\bmod d)$ for all $j$. Since $z_{j} \geqslant 0$ and $z_{j}^{\prime} \in\{0, \ldots, d-1\}$, this implies $z_{j} \geqslant z_{j}^{\prime}$. This together with (4) implies that in fact $z_{j}=z_{j}^{\prime}$ for all $j$, and thus $\mathbf{z}=\mathbf{z}^{\prime}$.

Since $I \mathbf{z}=I \mathbf{z}^{\prime}$ and $J \mathbf{x}^{(i)}=J \mathbf{x}^{\prime(i)}(i=2 \ldots d)$, linear combinations of Equations (1) and (2) imply that $B \mathbf{x}^{(i)}=B \mathbf{x}^{\prime(i)}$ for each $i=1 \ldots d$. By inductive assumption on $B$, this implies that $\mathbf{x}^{(i)}=\mathbf{x}^{\prime(i)}$ and hence $\mathbf{u}=\mathbf{v}$.

We thus obtain $\{0,1\}$-matrices $M_{i}$ with $k_{i}$ rows and $m_{i}$ columns such that

$$
k_{1}=m_{1}=d \quad \text { and } \quad k_{i+1}=d k_{i}+d \quad \text { and } \quad m_{i+1}=d m_{i}+k_{i} .
$$

Using a straightforward induction, we can check the following explicit formulas for $k_{i}$ and $m_{i}$ :

$$
k_{i}=\frac{d\left(d^{i}-1\right)}{d-1} \quad \text { and } \quad m_{i}=\frac{d}{(d-1)^{2}}\left[d^{i-1}((i-1)(d-1)+d(d-2))+1\right] .
$$

Hence, $k_{i} \leqslant \frac{d \cdot d^{i}}{d-1}, m_{i} \geqslant \frac{(i-1) \cdot d^{i}}{d-1}$, and $\log _{d}\left(m_{i}\right) \leqslant(i-1)(1+o(1))$, implying $k_{i} \leqslant \frac{m_{i} \cdot d}{\log _{d} m_{i}}(1+o(1))$. To interpolate between $m_{i}$ and $m_{i+1}$, one can join several of the constructed matrices into a 
block-diagonal matrix. Formally, if $f(m)$ denotes the least $k$ such that a $k \times m$ matrix as in the preceding exists, then $f\left(m+m^{\prime}\right) \leqslant f(m)+f\left(m^{\prime}\right)$ and $f\left(m_{i}\right) \leqslant \frac{m_{i} \cdot d}{\log _{d} m_{i}}(1+o(1))$. Standard methods then allow us to show that $f(m) \leqslant \frac{m \cdot d}{\log _{d} m}(1+o(1))$ (e.g., see Cantor and Mills [5, Theorem 2]; also see Martirosyan and Khachatryan [34] for a slightly more explicit construction for all $m$ ).

We remark that the bounds in Theorem 5 and Lemma 6 were also shown to be tight. Lemma 7 gives matrices that are also $d$-detecting, and hence the bound is tight for $d=2$ (and tight up to an $O(d)$ factor in general).

Note also that we can relax the non-negativity constraint to requiring that $\mathbf{u} \in \mathbb{Z}^{m}$ is any integer with all entries lower bounded by $-\left\lfloor\frac{d}{2}\right\rfloor$ and $\mathbf{v} \in\left\{-\left\lfloor\frac{d}{2}\right\rfloor, \ldots,\left\lfloor\frac{d}{2}\right\rfloor\right\}^{m}$. This is because $M \mathbf{u}=M \mathbf{v}$ is equivalent to $M(\mathbf{u}+\mathbf{c})=M(\mathbf{v}+\mathbf{c})$, where $\mathbf{c}$ is the constant $\left\lfloor\frac{d}{2}\right\rfloor$ vector. This allows to use the same detecting matrix for such pairs of vectors as well. However, note that some lower bound on the coefficients of $\mathbf{u}$ is necessary, since even if we fix $\mathbf{v}=0$, the matrix $M$ has a non-trivial kernel, giving many non-zero vectors $\mathbf{u} \in \mathbb{Z}^{m}$ satisfying $M \mathbf{u}=M \mathbf{v}$.

\subsection{Coefficient Reduction}

In further constructions, we will need a way to reduce coefficients in a given ILP FEAsiBILITY instance with a non-negative constraint matrix $A$ to $\{0,1\}$. We now prove that this can be done in a standard way by replacing each constraint with $O\left(\log \|A\|_{\infty}\right)$ constraints that check the original equality bit by bit. Here and throughout this article, we use the convention that for a vector $\mathbf{x}$, by $x_{i}$ we denote the $i$-th entry of $\mathbf{x}$.

Lemma 8 (Coefficient Reduction). Consider an instance $\{A \mathbf{x}=\mathbf{b}, \mathbf{x} \geqslant 0\}$ of ILP Feasibility, where $\mathbf{b} \in \mathbb{N}^{k}$ and $A$ is a non-negative integer matrix with $k$ rows and $\ell$ columns. In polynomial time, this instance can be reduced to an equivalent instance $\left\{A^{\prime} \mathbf{x}=\mathbf{b}^{\prime}, \mathbf{x} \geqslant 0\right\}$ of ILP FEASIBILITY, where $A^{\prime}$ is a $\{0,1\}$-matrix with $k^{\prime}=O\left(k \log \|A\|_{\infty}\right)$ rows and $\ell^{\prime}=\ell+O\left(k \log \|A\|_{\infty}\right)$ columns, and $\mathbf{b}^{\prime} \in \mathbb{N}^{k^{\prime}}$ is a vector with $\left\|\mathbf{b}^{\prime}\right\|_{\infty}=O\left(\|\mathbf{b}\|_{\infty}\right)$.

Proof. Denote $\delta=\left\lceil\log \left(1+\|A\|_{\infty}\right)\right\rceil=O\left(\log \|A\|_{\infty}\right)$. Consider a single constraint $\mathbf{a}^{\top} \mathbf{x}=b$, where $\mathbf{a} \in \mathbb{N}^{\ell}$ is a row of $A$ and $b \in \mathbb{N}$ is an entry of b. Let $a_{i}[j]$ be the $j$-th bit of $a_{i}$, the $i$-th entry of vector $\mathbf{a}$; similarly for $b$. By choice of $\delta$, $\|\mathbf{a}\|_{\infty} \leqslant 2^{\delta}-1$, so each entry of a has up to $\delta$ binary digits. Now, for $\mathbf{x} \in \mathbb{Z}^{n}$, the constraint $\mathbf{a}^{\top} \mathbf{x}=b$ is equivalent to

$$
\sum_{j=0}^{\delta-1} 2^{j}\left(\sum_{i=1}^{n} a_{i}[j] \cdot x_{i}\right)=b
$$

We rewrite this equation into $\delta$ equations, each responsible for verifying one bit. For this, we introduce $\delta-1$ carry variables $y_{0}, y_{1}, \ldots, y_{\delta-2}$ and emulate the standard algorithm for adding binary numbers by writing equations

$$
y_{j-1}+\sum_{i=1}^{n} a_{i}[j] \cdot x_{i}=b[j]+2 y_{j} \quad \text { for } j=0, \ldots, \delta-1,
$$

where $y_{-1}$ and $y_{\delta-1}$ are replaced with 0 , and $b[\delta-1]$ is replaced with the number whose binary digits are (from the least significant): $b[\delta-1], b[\delta], b[\delta+1], \ldots$ (we do this because $b$ may have more than $\delta$ digits). To get rid of the variable $y_{j}$ on the right-hand side, we let $B=2^{\lceil\log b\rceil}$ and 
introduce two new variables $y_{j}^{\prime}, y_{j}^{\prime \prime}$ for each carry variable $y_{j}$, with constraints

$$
y_{j}+y_{j}^{\prime}=B \quad \text { and } \quad y_{j}+y_{j}^{\prime \prime}=B \quad \text { for } j=0, \ldots, \delta-2,
$$

which is equivalent to $y_{j}^{\prime}=y_{j}^{\prime \prime}=B-y_{j}$. Hence, the previous equations can be replaced by

$$
y_{j-1}+\sum_{i=1}^{n} a_{i}[j] \cdot x_{i}+y_{j}^{\prime}+y_{j}^{\prime \prime}=b[j]+2 B \quad \text { for } j=0, \ldots, \delta-1 .
$$

We thus replace each row of $A$ with $2(\delta-1)+\delta$ rows and $3(\delta-1)$ auxiliary variables.

\subsection{Proof of Theorem 1}

ETH states that for some $c>0$, 3SAT with $n$ variables cannot be solved in time $O^{\star}\left(2^{c n}\right)$ (the $O^{\star}$ notation hides polynomial factors). It was introduced by Impagliazzio et al. [22] and developed by Impagliazzo and Paturi [21] to become a central conjecture for proving tight lower bounds for the complexity of various problems. Although the original statement considers the parameterization by the number of variables, the sparsification lemma [21] allows us to assume that the number of clauses is linear in the number of variables, and hence we have the following.

Theorem 9 (e.g., see Cygan et Al. [9, Theorem 14.4]). Unless ETH fails, there is no algorithm for 3SAT that runs in time $2^{o(n+m)}$, where $n$ and $m$ denote the numbers of variables and clauses.

We now proceed to the proof of Theorem 1.

Theorem 1 (RepeAted). Assuming ETH, there is no algorithm solving ILP FEASIBILITY instances $\{A \mathbf{x}=\mathbf{b}, \mathbf{x} \geqslant 0\}$ with $A \in\{0,1\}^{k \times \ell}, \mathbf{b} \in \mathbb{N}^{k}$, and $\ell,\|\mathbf{b}\|_{\infty}=O(k \log k)$ in time $2^{o(k \log k)}$.

Our first step is to decrease the number of occurrences of each variable. The (3,4)SAT problem is a variant of 3SAT where each clause uses exactly three different variables and every variable occurs in at most four clauses. Tovey [37] gave a linear reduction from 3SAT to (3,4)SAT-that is, an algorithm that, given an instance of 3SAT with $n$ variables and $m$ clauses, in linear time constructs an equivalent instance of $(3,4) \mathrm{SAT}$ with $O(n+m)$ variables and clauses. In combination with Theorem 9, this yields the following.

COROLLARY 10. Unless ETH fails, there is no algorithm for $(3,4) S A T$ that runs in time $2^{o(n+m)}$, where $n$ and $m$ denote the numbers of variables and clauses, respectively.

We now reduce $(3,4)$ SAT to ILP FeAsibility. A $(3,4)$ SAT instance $\varphi$ with $n$ variables and $m$ clauses can be encoded in a standard way as an ILP FEAsIBILITY instance with $O(n+m)$ variables and constraints as follows. For each formula variable $v$, we introduce two ILP variables $x_{v}$ and $x_{\neg v}$ with a constraint $x_{v}+x_{\neg v}=1$ (hence, exactly one of them should be 1 , the other 0 ). For each clause $c$, we introduce two auxiliary slack variables $y_{c}, z_{c}$ and two constraints: $y_{c}+z_{c}=2$ and $x_{\ell_{1}}+x_{\ell_{2}}+x_{\ell_{3}}+y_{c}=3$, where $\ell_{1}, \ell_{2}, \ell_{3}$ are the three literals in $c$. Since $y_{c}, z_{c}$ will not appear in any other constraints, the first constraint is equivalent to ensuring that $y_{c} \leqslant 2$, so the second constraint is equivalent to $x_{\ell_{1}}+x_{\ell_{2}}+x_{\ell_{3}} \geqslant 1$. This way, one can reduce in polynomial time a $(3,4)$ SAT instance $\varphi$ with $n$ variables and $m$ clauses into an equivalent instance $\left\{\mathbf{x} \in \mathbb{Z}^{\ell} \mid A \mathbf{x}=\right.$ $\mathbf{b}, \mathbf{x} \geqslant 0\}$ of ILP FEASIBILITY where

- the constraint matrix $A$ has $k:=n+2 m$ rows and $\ell:=2 n+2 m$ columns;

- each entry in $A$ is 0 or 1 ; 
- each row and column of $A$ contains at most four non-zero entries; and

- the target vector $\mathbf{b}$ has all entries equal to 1,2 , or 3 .

We now reduce the obtained instance to another ILP FEAsIBILITy instance containing only $O((n+m) / \log (n+m))$ constraints. Let $M$ be the detecting matrix given by Lemma 7 for $d=4$ and the required number of columns ( $m$ in the notation of the statement of Lemma 7) equal to the number or rows (constraints) in $A$, which is $k$. Then for any $\mathbf{x} \in \mathbb{N}^{\ell}$, we have $A \mathbf{x} \in \mathbb{N}^{k}$ (since $A$ is non-negative) and $\mathbf{b} \in\{0, \ldots, d-1\}^{k}$, hence by Lemma 7 we have that $A \mathbf{x}=\mathbf{b}$ if and only if $M A \mathbf{x}=M \mathbf{b}$. We conclude that the ILP FeAsibility instance $\left\{\mathbf{x} \in \mathbb{Z}^{\ell} \mid A^{\prime} \mathbf{x}=\mathbf{b}^{\prime}, \mathbf{x} \geqslant 0\right\}$ with $A^{\prime}=M A$ and $\mathbf{b}^{\prime}=M \mathbf{b}$ is equivalent to the previous instance $\left\{\mathbf{x} \in \mathbb{Z}^{\ell} \mid A \mathbf{x}=\mathbf{b}, \mathbf{x} \geqslant 0\right\}$.

The new instance has the same number $\ell^{\prime}=\ell=2 n+2 m$ of variables, but only $k^{\prime}=$ $O(k / \log k)=O((n+m) / \log (n+m))$ constraints. The entries of $\mathbf{b}^{\prime}=M \mathbf{b}$ are non-negative and bounded by $k \cdot\|\mathbf{b}\|_{\infty}=O(n+m)$. Similarly, entries of $A^{\prime}=M A$ are non-negative, and since every column of $A$ has at most four non-zero entries, we get $\left\|A^{\prime}\right\|_{\infty} \leqslant 4$.

To further reduce $\left\|A^{\prime}\right\|_{\infty}$, we apply Lemma 8 , replacing each row of $A^{\prime}$ by a constant number of $\{0,1\}$-rows and auxiliary variables. This way, we reduced in polynomial time a $(3,4)$ SAT instance $\varphi$ with $n$ variables and $m$ clauses into an equivalent ILP FeAsIBILITy instance $\{\mathbf{x} \in$ $\left.\mathbb{Z}^{\ell^{\prime \prime}} \mid A^{\prime \prime} \mathbf{x}=\mathbf{b}^{\prime \prime}, \mathbf{x} \geqslant 0\right\}$, where $A^{\prime \prime}$ is a $\{0,1\}$-matrix with $\ell^{\prime \prime}=\ell^{\prime}+O\left(k^{\prime}\right)=O(n+m)$ columns and $k^{\prime \prime}=\Theta\left(k^{\prime}\right)=\Theta((n+m) / \log (n+m))$ rows, whereas $\left\|\mathbf{b}^{\prime \prime}\right\|_{\infty}=O(n+m)$. Hence, $\ell^{\prime \prime},\left\|\mathbf{b}^{\prime \prime}\right\|_{\infty}=$ $O\left(k^{\prime \prime} \log k^{\prime \prime}\right)$.

We are now in position to finish the proof of Theorem 1. Suppose there is an algorithm for ILP FeAsibility that works in time $2^{o\left(k^{\prime \prime} \log k^{\prime \prime}\right)}$ on instances with $A \in\{0,1\}^{k^{\prime \prime} \times \ell^{\prime \prime}}$ and $\ell^{\prime \prime},\left\|\mathbf{b}^{\prime \prime}\right\|_{\infty}=$ $O\left(k^{\prime \prime} \log k^{\prime \prime}\right)$. Then applying the preceding reduction would solve $(3,4)$ SAT instances with $N=$ $n+m$ variables and clauses in time $2^{o((N / \log N) \cdot \log (N / \log N))}=2^{o(N)}$, which contradicts ETH by Corollary 10. This concludes the proof of Theorem 1.

\subsection{Reducing Coefficients in the Target Vector}

We now prove Corollary 2. In other words, we show that in Theorem 1, the coefficients in the target vector $\mathbf{b}$ can be reduced to constant, at the cost of introducing negative $(-1)$ coefficients in the matrix $A$.

Corollary 2 (REPEATED). Assuming ETH, there is no algorithm solving ILP FEASIBILITY instances $\{A \mathbf{x}=\mathbf{b}, \mathbf{x} \geqslant 0\}$ with $A \in\{-1,0,1\}^{k \times \ell}, \mathbf{b} \in\{0,1\}^{k}$, and $\ell=O(k \log k)$ in time $2^{o(k \log k)}$.

Proof of Corollary 2. Let $\{A \mathbf{x}=\mathbf{b}, \mathbf{x} \geqslant 0\}$ be an instance given by Theorem 1 , with $\ell,\|\mathbf{b}\|_{\infty}=$ $O(k \log k)$. Let $s:=\left\lceil\log \left(\|\mathbf{b}\|_{\infty}+1\right)\right\rceil$. To the system of linear equalities, we add $s+1$ new variables $z, y_{0}, \ldots, y_{s-1}$, with constraints $z=1$ and

$$
z+y_{0}+\cdots+y_{i-1}=y_{i} \quad \forall i=0, \ldots, s-1,
$$

which force $z=1$ and $y_{i}=2^{i}$. Then each original constraint $\mathbf{a}^{\top} \mathbf{x}=b$, where $\mathbf{a} \in \mathbb{N}^{\ell}$ is a row of $A$ and $b \in \mathbb{N}$ is an entry of $\mathbf{b}$, can be replaced by the constraint $\mathbf{a}^{\top} \mathbf{x}-\mathbf{c}^{\top} \mathbf{y}=0$, where $\mathbf{c}$ is chosen so that $\mathbf{c}^{\top} \mathbf{y}=b$-that is, the $i$-th entry of $\mathbf{c}$ is 0 or 1 depending on the $i$-th bit of $b$.

In matrix form, we thus created the following instance (where $B \in\{-1,0\}^{(1+s) \times \ell}$ is the matrix corresponding to the binary encoding of entries of $\mathbf{b}$, with the first column zero, since it corresponds to the variable $z$ ): 


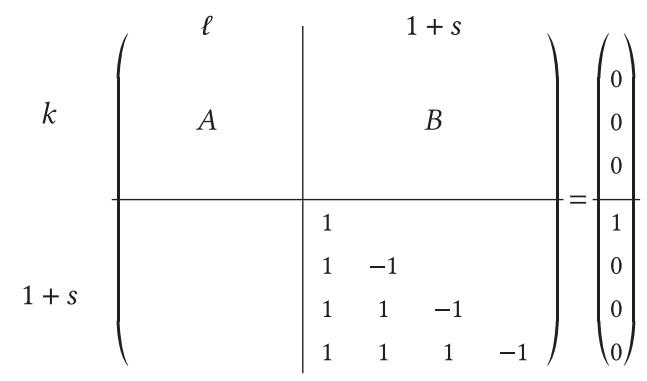

Since $s=O(\log k)$, the resulting matrix has $\{-1,0,1\}$ entries, $k+1+s=\Theta(k)$ rows, and $\ell+1+$ $s=O(k \log k)$ columns. The new target vector has only $\{0,1\}$ entries, as required.

\section{PARAMETERIZATION BY THE DUAL TREEDEPTH}

\subsection{Preliminaries}

Treedepth and dual treedepth. For a graph $G$, the treedepth of $G$, denoted $\operatorname{td}(G)$, can be defined recursively as follows:

$$
\operatorname{td}(G)= \begin{cases}1 & \text { if } G \text { has one vertex; } \\ \max \left(\operatorname{td}\left(G_{1}\right), \ldots, \operatorname{td}\left(G_{p}\right)\right) & \text { if } G \text { is disconnected and } G_{1}, \ldots, G_{p} \\ 1+\min _{u \in V(G)} \operatorname{td}(G-u) & \text { are its connected components; } \\ \text { if } G \text { has more than one vertex } & \text { and is connected. }\end{cases}
$$

See, for example, Nešetřil and Ossona de Mendez [35]. Equivalently, treedepth is the smallest possible height of a rooted forest $F$ on the same vertex set as $G$ such that whenever $u v$ is an edge in $G$, then $u$ is an ancestor of $v$ in $F$ or vice versa.

Since we focus on constraints, we consider, for a matrix $A$, the constraint graph or dual graph $G_{D}(A)$, defined as the graph with rows of $A$ as vertices where two rows are adjacent if and only if in some column they simultaneously contain a non-zero entry. The dual treedepth of $A$, denoted $\operatorname{td}_{D}(A)$, is the treedepth of $G_{D}(A)$.

The recursive definition (5) is elegantly reinterpreted in terms of row removals and partitioning into blocks as follows. A matrix $A$ is block decomposable if after permuting its rows and columns it can be presented in block-diagonal form-that is, rows and columns can be partitioned into intervals $R_{1}, \ldots, R_{p}$ and $C_{1}, \ldots, C_{p}$, for some $p \geqslant 2$, such that non-zero entries appear only in blocks $B_{1}, \ldots, B_{p}$, where $B_{i}$ is the block of entries at intersections of rows from $R_{i}$ with columns from $C_{i}$. It is easy to see that $A$ is block decomposable if and only if $G_{D}(A)$ is disconnected, and the finest block decomposition of $A$ corresponds to the partition of $G_{D}(A)$ into connected components. The blocks $B_{1}, \ldots, B_{p}$ in this finest partition are called the block components of $A$-they are not block decomposable. Then the recursive definition of treedepth provided in (5) translates to the following definition of the dual treedepth of a matrix $A$ :

$$
\operatorname{td}_{D}(A)= \begin{cases}1 & \text { if } A \text { has one row; } \\ \max \left(\operatorname{td}_{D}\left(B_{1}\right), \ldots, \operatorname{td}_{D}\left(B_{p}\right)\right) & \text { if } A \text { is block-decomposable and } \\ 1+\min _{\mathrm{a}^{\top} \text { :rows of } A} \operatorname{td}_{D}\left(A \backslash \mathbf{a}^{\top}\right) & \text { if } A \text { has more than one row and } \\ \text { is not block decomposable. }\end{cases}
$$

Here, $A \backslash \mathbf{a}^{\top}$ is the matrix obtained from $A$ by removing the row $\mathbf{a}^{\top}$. Intuitively, dual treedepth formalizes the idea that a block-decomposable matrix is as hard as the hardest of its block components, and that adding a single row makes it a bit harder, but not uncontrollably so. 
Graver bases. Two integer vectors $\mathbf{a}, \mathbf{b} \in \mathbb{Z}^{n}$ are sign compatible if $a_{i} \cdot b_{i} \geqslant 0$ for all $i=1, \ldots, n$. For $\mathbf{a}, \mathbf{b} \in \mathbb{Z}^{n}$, we write $\mathbf{a} \sqsubseteq \mathbf{b}$ if $\mathbf{a}$ and $\mathbf{b}$ are sign compatible and $\left|a_{i}\right| \leqslant\left|b_{i}\right|$ for all $i=1, \ldots, n$. Then, $\sqsubseteq$ is a partial order on $\mathbb{Z}^{n}$; we call it the conformal order. Note that $\sqsubseteq$ has a unique minimum element, which is the zero vector $\mathbf{0}$. We write $\sqsubset$ for the corresponding strict order $(\mathbf{a} \sqsubset \mathbf{b}$ if $\mathbf{a} \sqsubseteq \mathbf{b}$ and $\mathbf{a} \neq \mathbf{b}$ ).

For a matrix $A$, the Graver basis of $A$, denoted $\mathcal{G}(A)$, is the set of conformally minimal vectors in $\left(\operatorname{ker} A \cap \mathbb{Z}^{n}\right)-\{0\}$. It is easy to see by Dickson's lemma that $\left(\mathbb{Z}^{n}, \sqsubseteq\right)$ is a well quasi-ordering, and hence there are no infinite antichains with respect to the conformal order. It follows that the Graver basis of every matrix is finite, although it can be quite large. For a matrix $A$ and $p \in[1, \infty]$, we denote $g_{p}(A)=\max _{\mathbf{u} \in \mathcal{G}(A)}\|\mathbf{u}\|_{p}$.

\subsection{Upper Bound}

We start with the upper bound for the dual treedepth parameterization-that is, Theorem 3.

Theorem 3 (Repeated). There is an algorithm that solves any given ILP Optimization instance

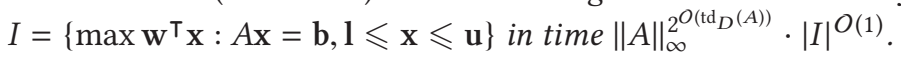

As explained in Section 1, this result easily follows from the work of Koutecký et al. [30] and the following lemma bounding $g_{1}(A)$ in terms of $\operatorname{td}_{D}(A)$ and $\|A\|_{\infty}$, for any integer matrix $A$.

LEMMA 11. For any matrix $A$ with integer entries, it holds that

$$
g_{1}(A) \leqslant\left(2\|A\|_{\infty}+1\right)^{2^{\mathrm{td}_{D}(A)}-1} .
$$

Before we prove Lemma 11, let us sketch how using the reasoning from Koutecký et al. [30] one can derive Theorem 3. Full details can be found in Eisenbrand et al. [13, Lemma 23], which also applies to non-linear objectives. Using the bound on the $\ell_{1}$-norm of vectors in the Graver basis of $A$, we can construct a $\Lambda$-Graver-best oracle for the considered ILP OptimizATION instance. This is an oracle that, given any feasible solution $\mathbf{x}$, returns another feasible solution $\mathbf{x}^{\prime}$ that differs from $\mathbf{x}$ only by an integer multiple not larger than $\Lambda$ of a vector from the Graver basis of $A$, and among such solutions achieves the best goal value of $\mathbf{w}^{\top} \mathbf{x}^{\prime}$. Such a $\Lambda$-Graver-best oracle runs in time $\left(\|A\|_{\infty} \cdot g_{1}(A)\right)^{O\left(\operatorname{tw}_{D}(A)\right)} \cdot|I|^{O(1)}$, where $\operatorname{tw}_{D}(A)$ is the treewidth of the constraint graph $G_{D}(A)$, which is always upper bounded by $\operatorname{td}_{D}(A)+1$. See the proof of Lemma 25 and the beginning of the proof of Theorem 3 in Koutecký et al. [30]; the reasoning there is explained in the context of tree-fold ILPs, but it uses only boundedness of the dual treedepth of $A$. Once a $\Lambda$-Graver-best oracle is implemented, we can use it to implement a Graver-best oracle (Lemma 14 in Koutecký et al. [30]) within the same asymptotic running time, and finally use the main theorem-Theorem 1 in Koutecký et al. [30]-to obtain the algorithm promised in Theorem 3.

We now proceed to the proof of Lemma 11.

Proof of Lemma 11. We proceed by induction on the number of rows of $A$ using the recursive definition (6). For the base case-when $A$ has one row-we may use the following well-known bound.

Claim 1 (Lemma 3.5.7 in De Loera et AL. [33]). If $A$ is an integer matrix with one row, then

$$
g_{1}(A) \leqslant 2\|A\|_{\infty}+1 \text {. }
$$

We note that the original bound of $2\|A\|_{\infty}-1$, stated in De Loera et al. [33], works only for non-zero $A$.

We now move to the induction step, so suppose the considered matrix $A$ has more than one row. We consider two cases: either $A$ is block decomposable or it is not. 
First suppose that $A$ is block decomposable. Let $B_{1}, \ldots, B_{p}$ be the block components of $A$, and let $R_{1}, \ldots, R_{p}$ and $C_{1}, \ldots, C_{p}$ be the corresponding partitions of rows and columns of $A$ into segments, respectively. Observe that integer vectors $\mathbf{u}$ from $\operatorname{ker} A$ are exactly vectors of the form $\left(\mathbf{v}^{(1)}\left|\mathbf{v}^{(2)}\right| \ldots \mid \mathbf{v}^{(p)}\right)$, where each $\mathbf{v}^{(i)}$ is an integer vector of length $\left|C_{i}\right|$ that belongs to $\operatorname{ker} B_{i}$. It follows that $\mathcal{G}(A)$ consists of vectors of the following form: for some $i \in\{1, \ldots, p\}$, put a vector from $\mathcal{G}\left(B_{i}\right)$ on coordinates corresponding to the columns of $C_{i}$, and fill all of the other entries with zeroes. Consequently, we have

$$
g_{1}(A) \leqslant \max _{i=1, \ldots, p} g_{1}\left(B_{i}\right)
$$

However, by (6), we have

$$
\operatorname{td}_{D}(A)=\max _{i=1, \ldots, p} \operatorname{td}_{D}\left(B_{i}\right) .
$$

Since each matrix $B_{i}$ has fewer rows than $A$, we may apply the induction assumption to matrices $B_{1}, \ldots, B_{p}$, thus inferring by (7) and (8) that

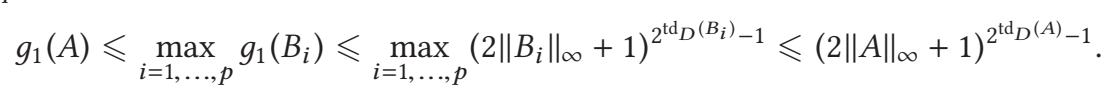

We are left with the case when $A$ is not block decomposable. For this, we use the following claim, which is essentially Lemma 3.7.6 and Corollary 3.7.7 in De Loera et al. [33]. The statement there is slightly different, but the same proof in fact proves the following bound.

Claim 2. Let $A$ be an integer matrix, and let $\mathbf{a}^{\top}$ be a row of $A$. Then

$$
g_{1}(A) \leqslant\left(2\left\|\mathbf{a}^{\top}\right\|_{\infty}+1\right) \cdot g_{1}\left(A \backslash \mathbf{a}^{\top}\right) \cdot g_{\infty}\left(A \backslash \mathbf{a}^{\top}\right) .
$$

Proof. Denote $B=A \backslash \mathbf{a}^{\top}$. Consider any vector $\mathbf{u} \in \mathcal{G}(A)$. Then $\mathbf{u}$ is also in $\operatorname{ker} B \cap \mathbb{Z}^{n}$, where $n$ is the number of columns of $A$. This means that we can write $\mathbf{u}$ as a sign-compatible sum of elements of the Graver basis of $B$-that is,

$$
\mathbf{u}=\sum_{i=1}^{p} \lambda_{i} \mathbf{g}_{i}
$$

for some $\lambda_{1}, \ldots, \lambda_{p} \in \mathbb{N}$ and distinct sign-compatible vectors $\mathbf{g}_{1}, \ldots, \mathbf{g}_{p} \in \mathcal{G}(B)$ (to see this, simply take a conformally minimal vector $\mathbf{g}_{1} \sqsubseteq \mathbf{u}$ in $\mathcal{G}(B)$ and repeat with $\mathbf{u}-\mathbf{g}_{1}$ ).

Let $\lambda$ be a vector of length $p$ with entries $\lambda_{1}, \ldots, \lambda_{p}$. Further, let $\mathbf{b}$ be also a vector of length $p$, where $b_{i}=\mathbf{a}^{\top} \mathbf{g}_{i}$. Considering $\mathbf{b}^{\top}$ as a matrix with one row, we have $\lambda \in \operatorname{ker} \mathbf{b}^{\top}$. Indeed, we have

$$
\sum_{i=1}^{p} \lambda_{i} b_{i}=\sum_{i=1}^{p} \lambda_{i}\left(\mathbf{a}^{\top} \mathbf{g}_{i}\right)=\mathbf{a}^{\top} \sum_{i=1}^{p} \lambda_{i} \mathbf{g}_{i}=\mathbf{a}^{\top} \mathbf{u}=0,
$$

because $\mathbf{u} \in \operatorname{ker} \mathbf{a}^{\top}$ due to $\mathbf{u} \in \mathcal{G}(A)$.

We now verify that in fact $\lambda \in \mathcal{G}\left(\mathbf{b}^{\top}\right)$. Indeed, since $\mathbf{u}$ is non-zero, $\lambda$ is non-zero as well. In addition, if there existed some non-zero $\lambda^{\prime} \sqsubset \lambda$ with $\lambda^{\prime} \in \operatorname{ker} \mathbf{b}^{\top}$, then the same computation as presented earlier would yield that $\mathbf{u}^{\prime}=\sum_{i=1}^{p} \lambda_{i}^{\prime} \mathbf{g}_{i}$ also belongs to $\operatorname{ker} A$. However, as vectors $\mathbf{g}_{1}, \ldots, \mathbf{g}_{p}$ are sign compatible, $\mathbf{0} \sqsubset \lambda^{\prime} \sqsubset \lambda$ would entail $\mathbf{0} \sqsubset \mathbf{u}^{\prime} \sqsubset \mathbf{u}$, a contradiction to the conformal minimality of $\mathbf{u}$ following from $\mathbf{u} \in \mathcal{G}(A)$.

Now that we know that $\lambda \in \mathcal{G}\left(\mathbf{b}^{\top}\right)$, we may use Claim 1 to infer that

$$
\|\lambda\|_{1} \leqslant 2\left\|\mathbf{b}^{\top}\right\|_{\infty}+1=2 \max _{i=1, \ldots, p}\left|\mathbf{a}^{\top} \mathbf{g}_{i}\right|+1 \leqslant 2\|\mathbf{a}\|_{\infty} \cdot g_{1}(B)+1 \leqslant\left(2\|\mathbf{a}\|_{\infty}+1\right) \cdot g_{1}(B) .
$$

Hence, we have

$$
\|\mathbf{u}\|_{1}=\left\|\sum_{i=1}^{p} \lambda_{i} \mathbf{g}_{i}\right\|_{1} \leqslant\|\lambda\|_{1} \cdot g_{\infty}(B) \leqslant\left(2\|\mathbf{a}\|_{\infty}+1\right) \cdot g_{1}(B) \cdot g_{\infty}(B) .
$$


This concludes the proof.

Suppose then that $A$ is not block decomposable. By (6), there exists a row $\mathbf{a}^{\top}$ of $A$ such that $\operatorname{td}_{D}\left(A \backslash \mathbf{a}^{\top}\right)=\operatorname{td}_{D}(A)-1$. Then, by Claim 2 and the inductive assumption, we have

$$
\begin{aligned}
& g_{1}(A) \leqslant\left(2\left\|\mathbf{a}^{\top}\right\|_{\infty}+1\right) \cdot g_{1}\left(A \backslash \mathbf{a}^{\top}\right) \cdot g_{\infty}\left(A \backslash \mathbf{a}^{\top}\right) \leqslant\left(2\|A\|_{\infty}+1\right) \cdot\left(g_{1}\left(A \backslash \mathbf{a}^{\top}\right)\right)^{2} \\
& \leqslant\left(2\|A\|_{\infty}+1\right)^{1+2 \cdot\left(2^{\text {td }_{D}(A)-1}-1\right)}=\left(2\|A\|_{\infty}+1\right)^{2^{\text {td }_{D}(A)}-1} .
\end{aligned}
$$

This concludes the proof.

\subsection{Lower Bound}

We now move to the proof of the lower bound, Theorem 4 .

THEOREM 4 (REPEATED). Assuming ETH, there is no algorithm that would solve any ILP FEASIBILITY

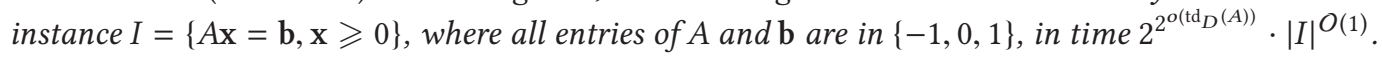

We will reduce from the SUBSET Sum problem: given non-negative integers $s_{1}, \ldots, s_{k}, t$, encoded in binary, decide whether there is a subset of numbers $s_{1}, \ldots, s_{k}$ that sums up to $t$. The standard NP-hardness reduction from 3SAT to SUBSET SUM takes an instance of 3SAT with $n$ variables and $m$ clauses, and produces an instance $\left(s_{1}, \ldots, s_{k}, t\right)$ of SUBSET Sum with a linear number of numbers and each of them of linear bit length-that is, $k \leqslant O(n+m)$ and $0 \leqslant s_{1}, \ldots, s_{k}, t<2^{\delta}$, for some $\delta \leqslant$ $O(n+m)$. See, for example, Abboud et al. [1] for an even finer reduction, yielding lower bounds for SubSET Sum under SETH. By Theorem 9, this immediately implies an ETH-based lower bound for Subset Sum.

LEMma 12. Unless ETH fails, there is no algorithm for SUBSET SUM that would solve any input instance $\left(s_{1}, \ldots, s_{k}, t\right)$ in time $2^{o(k+\delta)}$, where $\delta$ is the smallest integer such that $s_{1}, \ldots, s_{k}, t<2^{\delta}$.

The idea for our reduction from SubSET Sum to ILP FeAsibility is as follows. Given an instance $\left(s_{1}, \ldots, s_{k}, t\right)$, we first construct numbers $s_{1}, \ldots, s_{k}$ using ILPs $P_{1}, \ldots, P_{k}$, where each $P_{i}$ uses only constant-size coefficients and has dual treedepth $O(\log \delta)$. The ILP $P_{i}$ will have a designated variable $z_{i}$ and two feasible solutions: one that sets $z_{i}$ to 0 and one that sets it to $s_{i}$. Similarly, we can construct an ILP $Q$ that forces a designated variable $w$ to be set to $t$. Having that, the whole input instance can be encoded using one additional constraint: $z_{1}+\cdots+z_{k}-w=0$. To construct each $P_{i}$, we first create $\delta$ variables $y_{0}, y_{1}, \ldots, y_{\delta-1}$ that are either all evaluated to 0 or all evaluated to $2^{0}, 2^{1}, \ldots, 2^{\delta-1}$, respectively; this involves constraints of the form $y_{j+1}=2 y_{j}$. Then the number $s_{i}$ (or 0 ) can be obtained on a new variable $z_{i}$ using a single constraint that assembles the binary encoding of $s_{i}$. The crucial observation is that the constraint graph $G_{D}\left(P_{i}\right)$ consists of a path on $\delta$ vertices and one additional vertex, and thus has treedepth $O(\log \delta)$.

We start implementing this plan formally by giving the construction for a single number $s$.

Lemma 13. For all positive integers $\delta$ and $s$ satisfying $0 \leqslant s<2^{\delta}$, there exists an instance $P=$ $\{A \mathbf{x}=\mathbf{b}, \mathbf{x} \geqslant 0\}$ of ILP FEASIBILITY with the following properties:

- $A$ has all entries in $\{-1,0,1,2\}$ and $\operatorname{td}_{D}(A) \leqslant \log \delta+O(1)$;

- $\mathbf{b}$ is a vector with all entries in $\{0,1\}$; and

- $P$ has exactly two solutions $\mathbf{x}^{(1)}$ and $\mathbf{x}^{(2)}$, where $x_{1}^{(1)}=0$ and $x_{1}^{(2)}=s$.

Moreover, the instance $P$ can be constructed in time polynomial in $\delta+\log s$.

Proof. We shall use $n+2$ variables, denoted for convenience by $y_{0}, y_{1}, \ldots, y_{\delta-1}, z, u$; these are arranged into the variable vector $\mathbf{x}$ of length $\delta+2$ so that $x_{1}=z$. Letting $b_{0}, b_{1}, \ldots, b_{\delta-1}$ be the 
consecutive digits of the number $s$ in the binary encoding, the instance $P$ then looks as follows.

$$
\begin{aligned}
& u+y_{0}=1 \\
& 2 y_{0}-y_{1}=0
\end{aligned}
$$

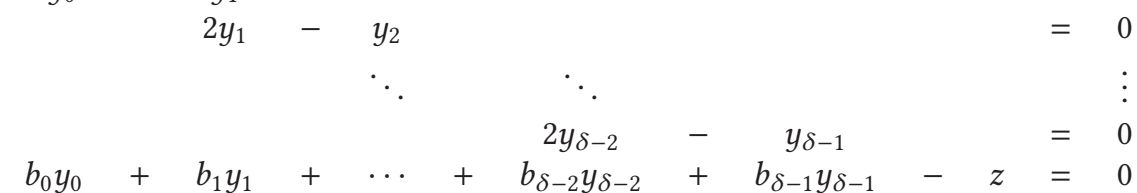

Since $0 \leqslant u \leqslant 1$, it is easy to see that $P$ has exactly two solutions in non-negative integers:

- If one sets $u=1$, then all of the other variables need to be set to 0 .

- If one sets $u=0$, then $y_{i}$ needs to be set to $2^{i}$ for all $i=0,1, \ldots, \delta-1$, and then $z$ needs to be set to $s$ by the last equation.

It remains to analyze the dual treedepth of $A$. Observe that the constraint graph $G_{D}(A)$ consists of a path of length $\delta$, plus one vertex corresponding to the last equation that may have an arbitrary neighborhood within the path. Since the path on $\delta$ vertices has treedepth $\lceil\log (\delta+1)\rceil$, it follows that $G_{D}(A)$ has treedepth at most $1+\lceil\log (\delta+1)\rceil \leqslant \log \delta+O(1)$.

We note that in the preceding construction, one may remove the variable $u$ and replace the constraint $u+y_{0}=1$ with $y_{0}=1$, thus forcing only one solution: the one that sets the first variable to $s$. This will be used later.

We are ready to show the core part of the reduction.

Lemma 14. An instance $\left(s_{1}, \ldots, s_{k}, t\right)$ of SUBSET SUM with $0 \leqslant s_{i}, t<2^{\delta}$ for $i=1, \ldots, k$, can be reduced in polynomial time to an equivalent instance $\{A \mathbf{x}=\mathbf{b}, \mathbf{x} \geqslant 0\}$ of ILP FEASIBILITY where entries of $A$ are in $\{-1,0,1,2\}$, entries of $\mathbf{b}$ are in $\{0,1\}$, and $\operatorname{td}_{D}(A) \leqslant \log \delta+O(1)$.

Proof. For each $i \in\{1, \ldots, k\}$, apply Lemma 13 to construct a suitable instance $P_{i}=\left\{A_{i} \mathbf{x}=\right.$ $\left.\mathbf{b}_{i}, \mathbf{x} \geqslant 0\right\}$ of ILP FEASIBILITY for $s=s_{i}$. In addition, apply Lemma 13 to construct a suitable instance $Q=\{C \mathbf{x}=\mathbf{d}, \mathbf{x} \geqslant 0\}$ of ILP FEAsibility for $s=t$, and modify it as explained after the lemma's proof so that there is only one solution, setting the first variable to $t$. Let

$$
A=\left(\begin{array}{ccccc} 
& & \mathbf{c}^{\top} & & \\
\hline A_{1} & & & & \\
& A_{2} & & & \\
& & \ddots & & \\
& & & A_{k} & \\
& & & & C
\end{array}\right)
$$

where

$$
\mathbf{c}^{\top}=(10 \ldots 0|10 \ldots 0| \ldots|10 \ldots 0|(-1) 0 \ldots 0)
$$

with consecutive blocks of lengths equal to the numbers of columns of $A_{1}, \ldots, A_{k}$, and $C$, respectively. Observe that

$$
\operatorname{td}_{D}(A) \leqslant 1+\max \left(\operatorname{td}_{D}\left(A_{1}\right), \ldots, \operatorname{td}_{D}\left(A_{k}\right), \operatorname{td}_{D}(C)\right)=\log \delta+O(1) .
$$

Further, let

$$
\mathbf{b}^{\top}=\left(0\left|\mathbf{b}_{1}^{\top}\right| \ldots\left|\mathbf{b}_{k}^{\top}\right| \mathbf{d}^{\top}\right)
$$

We now claim that the ILP $\{A \mathbf{x}=\mathbf{b}, \mathbf{x} \geqslant 0\}$ is feasible if and only if the input instance of SuBSET Sum has a solution. Indeed, if we denote by $z_{1}, \ldots, z_{k}, w$ the variables corresponding to the first columns of blocks $A_{1}, \ldots, A_{k}, C$, respectively, then by Lemma 13 within each block $A_{i}$ there are two 
ways of evaluating variables corresponding to columns of $A_{i}$ : one setting $z_{i}=0$ and the other setting $z_{i}=s_{i}$. However, there is only one way of evaluating the variables corresponding to columns of $C$, which sets $w=t$. The first row of $A$ then constitutes the constraint $z_{1}+\cdots+z_{k}-w=0$, which can be satisfied by setting $z_{i}$-s and $w$ as earlier if and only if some subset of the numbers $s_{1}, \ldots, s_{k}$ sums up to $t$.

It remains to reduce entries in $A$ equal to 2 , simply by duplicating variables.

LEMMA 15. An instance $\{A \mathbf{x}=\mathbf{b}, \mathbf{x} \geqslant 0\}$ of ILP FEASIBILITY where entries of $A$ are in $\{-1,0,1,2\}$ and entries of $\mathbf{b}$ are in $\{0,1\}$ can be reduced in polynomial time to an equivalent instance $\left\{A^{\prime} \mathbf{x}=\right.$ $\left.\mathbf{b}^{\prime}, \mathbf{x} \geqslant 0\right\}$ of ILP FEASIBILITY with all entries in $\{-1,0,1\}$ and $\operatorname{td}_{D}\left(A^{\prime}\right) \leqslant \operatorname{td}_{D}(A)+1$.

Proof. It suffices to duplicate each variable $x$ by introducing a variable $x^{\prime}$, adding a constraint $x-x^{\prime}=0$, and replacing occurrences of $2 x$ in constraints by $x+x^{\prime}$. In the dual graph, this results in introducing a new vertex (for the constraint $x-x^{\prime}=0$ ), adjacent only to those constraints that contained $x$, which form a clique in $G_{D}(A)$ (they are pairwise adjacent). The new vertices are nonadjacent to each other. We show that in total, this operation can only increase the dual treedepth by at most 1 .

Let $F$ be a rooted forest of height $\operatorname{td}\left(G_{D}(A)\right)$ with the same vertex set as $G_{D}(A)$ such that whenever $u v$ is an edge of $G_{D}(A)$, then $u$ is an ancestor of $v$ in $F$ or vice versa. Then in particular, for each original variable $x$, the constraints containing it form a clique in $G_{D}(A)$, so the constraint that is the lowest in $F$, say $\mathbf{a}^{\top}$, has all of the others as ancestors. This means that the new vertex representing the constraint $x-x^{\prime}=0$ can be added to $F$ as a pending leaf below $\mathbf{a}^{\top}$. Doing this for each original variable $x$ can only add pendant leaves to original vertices of $F$, which increases its height by at most 1 .

Theorem 4 now follows by observing that combining the reductions of Lemma 14 and Lemma 15 with a hypothetical algorithm for ILP FEASIBILITY on $\{-1,0,1\}$-input with running time $2^{2^{o\left(\mathrm{td}_{D}(A)\right)}}$. $|I|^{O(1)}$, or just $2^{o\left(2^{\mathrm{td}^{(A)}}\right)} \cdot|I|^{O(1)}$, would yield an algorithm for SubSET Sum with running time $2^{o(k+\delta)}$, contradicting ETH by Lemma 12 .

The proof is straightforward to adapt to larger values of $\|A\|_{\infty}$. Specifically, ETH and the proof of Lemma 12 imply that there is a constant $c>0$ such that SubSET Sum with $N$ numbers of magnitude $<2^{N}$ cannot be solved in time $2^{c N} \cdot N^{O(1)}$. Fix any function $\Delta: \mathbb{N} \rightarrow \mathbb{N}, \Delta \geqslant 2$ that is computable in polynomial time. In Lemma 13, one can replace the binary encoding with a $\Delta(N)$-ary encoding-that is, representing numbers of magnitude $<2^{N}$ as $\log _{\Delta(N)}\left(2^{N}\right)=\frac{N}{\log _{2} \Delta}$ digits between 0 and $\Delta(N)-1$. This results in an instance with $\|A\|_{\infty}=\Delta(N),\|\mathbf{b}\|_{\infty}=1$, and $\log _{2}\left(\frac{N}{\log _{2} \Delta}\right) \leqslant \operatorname{td}_{D}(A) \leqslant \log _{2}\left(\frac{N}{\log _{2} \Delta}\right)+C$, for some universal constant $C$; the outer logarithm is still binary, as it comes from the bound on the treedepth of a path. Afterward, Lemma 14 results in an instance of size polynomial in $N$ with similarly bounded parameters. Therefore, an algorithm solving such instances in time $\Delta^{c \cdot 2^{\text {td }} D^{-C}} \cdot|I|^{O(1)} \leqslant 2^{c N} \cdot N^{O(1)}$ would contradict ETH. Replacing $c$ with $c \cdot 2^{-C}$, we obtain the following theorem.

THEOREM 16. There are universal constants $c, C$ such that the following holds. Fix any poly-time computable $\Delta: \mathbb{N} \rightarrow \mathbb{N}, \Delta \geqslant 2$. If all ILP FeAsibility instances with $\|A\|_{\infty}=\Delta(N)$, $\|\mathbf{b}\|_{\infty}=1,|I|=$ $N^{\Theta(1)}$, and $\log _{2}\left(\frac{N}{\log _{2} \Delta}\right) \leqslant \operatorname{td}_{D}(A) \leqslant \log _{2}\left(\frac{N}{\log _{2} \Delta}\right)+C$ can be solved in time $\Delta^{c \cdot 2^{\mathrm{td} D}} \cdot|I|^{O(1)}$, then ETH is false.

In particular, the statement holds for any constant $\Delta(N)=\Delta$. On the other extreme, for any constant $t \in \mathbb{N}$, one can choose $\Delta(N)=2^{N / 2^{t}}$, resulting in $t \leqslant \operatorname{td}_{D}(A) \leqslant t+C$. 


\section{CONCLUSION}

We conclude this work by stating two concrete open problems in the topic.

First, apart from considering the standard form $\{A \mathbf{x}=\mathbf{b}, \mathbf{x} \geqslant 0\}$, Eisenbrand and Weismantel [14] also studied the more general setting of ILPs of the form $\{A \mathbf{x}=\mathbf{b}, \mathbf{l} \leqslant \mathbf{x} \leqslant \mathbf{u}\}$, where $\mathbf{l}$ and $\mathbf{u}$ are integer vectors. In other words, instead of only requiring that every variable is non-negative, we put an arbitrary lower and upper bound on the values it can take. Note that such lower and upper bounds can be easily emulated in the standard formulation using slack variables, but this would require adding more constraints to the matrix $A$; the key here is that we do not count these lower and upper bounds in the total number of constraints $k$. For this more general setting, Eisenbrand and Weismantel [14] gave an algorithm with running time $k^{O\left(k^{2}\right)} \cdot\|A\|_{\infty}^{O\left(k^{2}\right)} \cdot|I|^{O(1)}$, which boils down to $2^{O\left(k^{2} \log k\right)} \cdot|I|^{O(1)}$ when $\|A\|_{\infty}=O(1)$. (A typo leading to a $2^{O\left(k^{2}\right)} \cdot|I|^{O(1)}$ bound has been fixed in later versions of the work). Is this running time optimal, or could the $2^{O\left(k^{2} \log k\right)}$ factor be improved? Note that Theorem 1 implies a $2^{o(k \log k)}$-time lower bound, unless ETH fails.

Second, in this work, we studied the parameter dual treedepth of the constraint matrix $A$, but of course one can also consider the primal treedepth. It can be defined as the treedepth of the graph over the columns (variables) of $A$, where two columns are adjacent if they have a non-zero entry in same row (the variables appear simultaneously in some constraint). It is known that ILP FeAsibility and ILP Optimization are fixed-parameter tractable when parameterized by $\|A\|_{\infty}$ and $\operatorname{td}_{P}(A)$-that this, there is an algorithm with running time $f\left(\|A\|_{\infty}, \operatorname{td}_{P}(A)\right) \cdot|I|^{O(1)}$, for some function $f$ [13] (also see Eisenbrand et al. [12], Koutecký et al. [30], and a different method by Ganian and Ordyniak [17]). Again, the key ingredient here is an inequality on $\ell_{\infty}$-norms of the elements of the Graver basis of any integer matrix $A: g_{\infty}(A) \leqslant h\left(\|A\|_{\infty}, \operatorname{td}_{P}(A)\right)$ for some function $h$. The first bound on $g_{\infty}(A)$ was given by Aschenbrenner and Hemmecke [2]. The work of Aschenbrenner and Hemmecke [2] considers the setting of multi-stage stochastic programming (MSSP), which is related to primal treedepth in the same way as tree-fold ILPs are related to dual treedepth. The translation between MSSP and primal treedepth was first formulated by Koutecký et al. [30]. However, to establish a bound on $g_{\infty}(A)$, Aschenbrenner and Hemmecke [2] use the theory of well quasi-orderings (in a highly non-trivial way) and consequently give no direct bounds on the function $h$. Recently, Klein [26] gave the first constructive bound on $g_{\infty}(A)$ for MSSP. However, we conjecture that the function $h$ has to be non-elementary in $\operatorname{td}_{P}(A)$. If this were the case, an example could likely be used to prove a non-elementary lower bound under ETH for ILP FEASIBILITY under that $\operatorname{td}_{P}(A)$ parameterization (with $\|A\|_{\infty}=O(1)$ ).

\section{ACKNOWLEDGMENTS}

The authors would like to thank anonymous referees for their input.

\section{REFERENCES}

[1] Amir Abboud, Karl Bringmann, Danny Hermelin, and Dvir Shabtay. 2019. SETH-based lower bounds for subset sum and bicriteria path. In Proceedings of the 30th Annual ACM-SIAM Symposium on Discrete Algorithms (SODA'19). 41-57.

[2] Matthias Aschenbrenner and Raymond Hemmecke. 2007. Finiteness theorems in stochastic integer programming. Foundations of Computational Mathematics 7, 2 (2007), 183-227.

[3] Marthe Bonamy, Łukasz Kowalik, Michal Pilipczuk, Arkadiusz Socała, and Marcin Wrochna. 2019. Tight lower bounds for the complexity of multicoloring. ACM Transactions on Computation Theory 11, 3 (2019), Article 13, 19 pages. DOI : https://doi.org/10.1145/3313906

[4] Nader H. Bshouty. 2009. Optimal algorithms for the coin weighing problem with a spring scale. In Proceedings of the 22nd Conference on Learning Theory (COLT'09).

[5] David G. Cantor and W. H. Mills. 1966. Determination of a subset from certain combinatorial properties. Canadian Journal of Mathematics 18 (1966), 42-48. 
[6] Timothy Chan, Jacob W. Cooper, Martin Koutecký, Daniel Král, and Kristýna Pekárková. 2019. Optimal matrix treedepth and a row-invariant parameterized algorithm for integer programming. arXiv:1907.06688.

[7] Lin Chen. 2019. On block-structured integer programming and its applications. In Nonlinear Combinatorial Optimization, D.-Z. Du, P. M. Pardalow, and Z. Zhang (Eds.). Springer, Cham, 153-177. DOI : https://doi.org/10.1007/978-3-03016194-1_7

[8] Lin Chen and Dániel Marx. 2018. Covering a tree with rooted subtrees-Parameterized and approximation algorithms. In Proceedings of the 29th Annual ACM-SIAM Symposium on Discrete Algorithms (SODA'18). 2801-2820.

[9] Marek Cygan, Fedor V. Fomin, Łukasz Kowalik, Daniel Lokshtanov, Dániel Marx, Marcin Pilipczuk, Michał Pilipczuk, and Saket Saurabh. 2015. Parameterized Algorithms. Springer.

[10] Pavel Dvořák, Eduard Eiben, Robert Ganian, Dušan Knop, and Sebastian Ordyniak. 2017. Solving integer linear programs with a small number of global variables and constraints. In Proceedings of the 26th International foint Conference on Artificial Intelligence (IFCAI'17). 607-613. DOI : https://doi.org/10.24963/ijcai.2017/85

[11] Eduard Eiben, Robert Ganian, Dušan Knop, and Sebastian Ordyniak. 2018. Unary integer linear programming with structural restrictions. In Proceedings of the $26 \mathrm{~h}$ International foint Conference on Artificial Intelligence (IfCAI'18). 1284-1290. DOI : https://doi.org/10.24963/ijcai.2018/179

[12] Friedrich Eisenbrand, Christoph Hunkenschröder, and Kim-Manuel Klein. 2018. Faster algorithms for integer programs with block structure. In Proceedings of the 45th International Colloquium on Automata, Languages, and Programming (ICALP'18). Article 49, 13 pages.

[13] Friedrich Eisenbrand, Christoph Hunkenschröder, Kim-Manuel Klein, Martin Koutecký, Asaf Levin, and Shmuel Onn. 2019. An algorithmic theory of integer programming. arxiv:1904.01361.

[14] Friedrich Eisenbrand and Robert Weismantel. 2019. Proximity results and faster algorithms for integer programming using the Steinitz lemma. ACM Transactions on Algorithms 16, 1 (Nov. 2019). Article 5, 14 pages. DOI : https://doi.org/ $10.1145 / 3340322$

[15] Fedor V. Fomin, Fahad Panolan, M. S. Ramanujan, and Saket Saurabh. 2018. On the optimality of pseudo-polynomial algorithms for integer programming. arXiv:1607.05342.

[16] András Frank and Éva Tardos. 1987. An application of simultaneous diophantine approximation in combinatorial optimization. Combinatorica 7, 1 (1987), 49-65.

[17] Robert Ganian and Sebastian Ordyniak. 2018. The complexity landscape of decompositional parameters for ILP. Artificial Intelligence 257 (2018), 61-71. DOI : https://doi.org/10.1016/j.artint.2017.12.006

[18] Robert Ganian, Sebastian Ordyniak, and M. S. Ramanujan. 2017. Going beyond primal treewidth for (M)ILP. In Proceedings of the 31st AAAI Conference on Artificial Intelligence (AAAI'17). 815-821. http://aaai.org/ocs/index.php/AAAI/ AAAI17/paper/view/14272.

[19] Vladimir Grebinski and Gregory Kucherov. 2000. Optimal reconstruction of graphs under the additive model. Algorithmica 28, 1 (2000), 104-124.

[20] Raymond Hemmecke, Shmuel Onn, and Lyubov Romanchuk. 2013. $n$-Fold integer programming in cubic time. Mathematical Programming 137, 1-2 (2013), 325-341. DOI : https://doi.org/10.1007/s10107-011-0490-y

[21] Russell Impagliazzo and Ramamohan Paturi. 2001. On the complexity of $k$-SAT. Fournal of Computer and System Sciences 62, 2 (2001), 367-375.

[22] Russell Impagliazzo, Ramamohan Paturi, and Francis Zane. 2001. Which problems have strongly exponential complexity? Journal of Computer and System Sciences 63, 4 (2001), 512-530.

[23] Klaus Jansen, Kim-Manuel Klein, Marten Maack, and Malin Rau. 2019. Empowering the configuration-IP-New PTAS results for scheduling with setups times. In Proceedings of the 11th Innovations in Theoretical Computer Science (ITCS'19). Article 44, 19 pages.

[24] Klaus Jansen and Lars Rohwedder. 2019. On integer programming and convolution. In Proceedings of the 11th Innovations in Theoretical Computer Science (ITCS'19). Article 43, 17 pages. DOI : https://doi.org/10.4230/LIPIcs.ITCS.2019.43 arXiv:1803.04744

[25] Ravi Kannan. 1987. Minkowski’s convex body theorem and integer programming. Mathematics of Operations Research 12, 3 (1987), 415-440.

[26] Kim-Manuel Klein. 2020. About the complexity of two-stage stochastic IPs. In Integer Programming and Combinatorial Optimization. Lecture Notes in Computer Science, Vol. 12125. Springer, 252-265. DOI : https://doi.org/10.1007/978-3030-45771-6_20 arXiv:1901.01135

[27] Dušan Knop and Martin Koutecký. 2018. Scheduling meets $n$-fold integer programming. fournal of Scheduling 21, 5 (2018), 493-503.

[28] Dušan Knop, Martin Koutecký, and Matthias Mnich. 2017. Voting and bribing in single-exponential time. In Proceedings of the 34th International Symposium on Theoretical Aspects of Computer Science (STACS'17). Article 46, 14 pages. 
[29] Dušan Knop, Martin Koutecký, and Matthias Mnich. 2019. Combinatorial $n$-fold integer programming and applications. Mathematical Programming. Online. DOI : https://doi.org/10.1007/s10107-019-01402-2

[30] Martin Koutecký, Asaf Levin, and Shmuel Onn. 2018. A parameterized strongly polynomial algorithm for block structured integer programs. In Proceedings of the 45th International Colloquium on Automata, Languages, and Programming (ICALP'18). Article 85, 14 pages.

[31] Hendrik W. Lenstra. 1983. Integer programming with a fixed number of variables. Mathematics of Operations Research 8, 4 (1983), 538-548.

[32] Bernt Lindström. 1965. On a combinatorial problem in number theory. Canadian Mathematical Bulletin 8, 4 (1965), 477-490.

[33] Jesús A. De Loera, Raymond Hemmecke, and Matthias Köppe. 2013. Algebraic and Geometric Ideas in the Theory of Discrete Optimization. MOS-SIAM Series on Optimization, Vol. 14. SIAM.

[34] S. S. Martirosyan and G. G. Khachatryan. 1989. Construction of signature codes and the coin weighing problem. Problems of Information Transmission 25, 4 (1989), 96-97. In Russian.

[35] Jaroslav Nešetřil and Patrice Ossona de Mendez. 2012. Sparsity-Graphs, Structures, and Algorithms. Algorithms and Combinatorics, Vol. 28. Springer.

[36] Christos H. Papadimitriou. 1981. On the complexity of integer programming. Fournal of the ACM 28, 4 (1981), 765-768. DOI : https://doi.org/10.1145/322276.322287

[37] Craig A. Tovey. 1984. A simplified NP-complete satisfiability problem. Discrete Applied Mathematics 8, 1 (1984), 85-89.

Received July 2019; revised February 2020; accepted April 2020 